\title{
Influence of Torso Model Complexity on the Noninvasive Localization of Ectopic Ventricular Activity
}

\author{
Olena Punshchykova ${ }^{1}$, Jana Švehlíková2 ${ }^{2}$, Milan Tyšler ${ }^{1,2}$, Richard Grünes ${ }^{1}$, Ksenia Sedova ${ }^{1}$, \\ Pavel Osmančík ${ }^{3}$, Jana Žd'árská ${ }^{3}$, Dalibor Heřman ${ }^{3}$, Peter Kneppo ${ }^{1}$ \\ ${ }^{I}$ Department of Biomedical Technology, Faculty of Biomedical Engineering, Czech Technical University in Prague, \\ nám. Sítná 3105, 27201, Kladno, Czech Republic, olena.punshchykova@fbmi.cvut.cz \\ ${ }^{2}$ Institute of Measurement Science Slovak Academy of Sciences, Department of Biomeasurements, Dúbravská cesta, 9 , \\ 84104, Bratislava, Slovakia \\ ${ }^{3} 3 r d$ Faculty of Medicine, Charles University and Cardiocenter, University Hospital Královské Vinohrady, Šrobárova, 50, \\ 10034, Prague, Czech Republic
}

Location of premature ectopic ventricular activity was assessed noninvasively in five patients using integral body surface potential maps and inverse solution in terms of a single dipole. Precision of the inverse solution was studied using three different torso models: homogeneous torso model, inhomogeneous torso model including lungs and heart ventricles and inhomogeneous torso model including lungs, heart ventricles and atria, aorta and pulmonary artery. More stable results were obtained using the homogeneous model. However, in some patients the location of the resulting dipole representing the focus of ectopic activity was shifted between solutions using the homogeneous and inhomogeneous models. Comparison of solutions with inhomogeneous torso models did not show significantly different dispersions, but localization of the focus was better when a torso model including atria and arteries was used. The obtained results suggest that presented noninvasive localization of the ectopic focus can be used to shorten the time needed for successful ablation and to increase its success rate.

Keywords: Body surface potential mapping, ectopic activation, premature ventricular contraction, inhomogeneous torso model, inverse problem of electrocardiography.

\section{INTRODUCTION}

Premature ventricular complexes (PVCs) present an asymptomatic finding in many patients. However, especially if present in higher frequency, PVCs are associated with symptoms such as palpitations or fatigue. Frequent PVCs, defined as greater than $20 \%$ of all QRS complexes, can lead to the development of left ventricular dysfunction.

The origin of PVCs can be anywhere in the ventricles and adjacent structures with predominant location in the right and left outflow tracts (RVOT, LVOT). Several methods were developed to noninvasively predict the position of its focus. However, their accuracy is limited.

Generally, PVCs can be treated with anti-arrhythmic drugs. If PVCs are symptomatic or in higher frequency, and do not respond to pharmacological treatment, they are indicated for catheter radiofrequency ablation [1].

The ablation is an invasive procedure performed during the electrophysiological (EP) study in a catheter laboratory. The catheter is guided under X-ray control and optionally by a 3D navigational system to localize the PVC focus.
Accurate localization of the PVC focus is the most important factor for the success rate of the ablation that reached $77 \%$ in a long term follow-up [2]. However, duration of such procedure may be up to several hours. Therefore, it would be desirable to estimate the PVC origin noninvasively before the invasive EP study. This could significantly decrease the time of the ablation procedure and improve its success rate. Noninvasive localization in comparison with the invasive EP study does not introduce additional risks as bleeding, infection, damage of the vessel, perforation of the heart, etc.

In order to localize the PVC focus noninvasively, the inverse problem of electrocardiography should be solved. For its solution multiple ECG leads represented as body surface potential maps (BSPMs) and a realistic model of the patient's torso (based on a CT or MR scan) are used.

The aim of this study was to analyze the influence of the complexity of patient-specific torso model on the noninvasive localization of the PVC origin. The inverse problem was solved using equivalent cardiac generator represented by one dipole as proposed in [3], [4], [5]. 


\section{SUBJECT \& METHODS}

Two male (Pat004, 77 years and Pat006, 51 years) and three female patients (Pat005, 43 years, Pat007, 54 years, Pat009, 74 years) with frequent monomorphic PVCs underwent measurements of BSPMs, whole torso CT scanning and intracardiac EP study. All procedures were approved by the Ethic Committee of the University Hospital Kralovske Vinohrady, where the data were collected after obtaining written informed consent from the patients.

Multichannel ECG recording was performed by ProCardio-8 system [6]. ECG signals from limb leads and 96 chest leads organized in 12 strips of 8 leads evenly distributed over the patient's thorax were recorded using $\mathrm{Ag} / \mathrm{AgCl}$ electrodes with active adapters (Fig.1.A). Signals were sampled at $1 \mathrm{kHz}$ with 16-bit resolution; the record length was several minutes. Five ectopic beats from the multichannel ECG records of each patient were selected for further processing. In each ectopic beat, the initial $20 \mathrm{~ms}$ interval from the ventricular activation was selected to compute integral BSPMs [4].

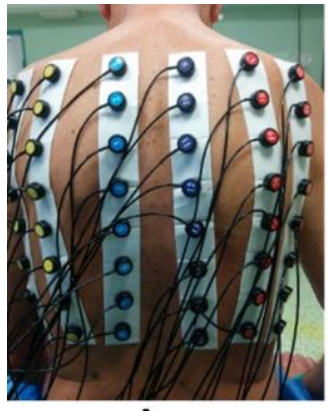

A

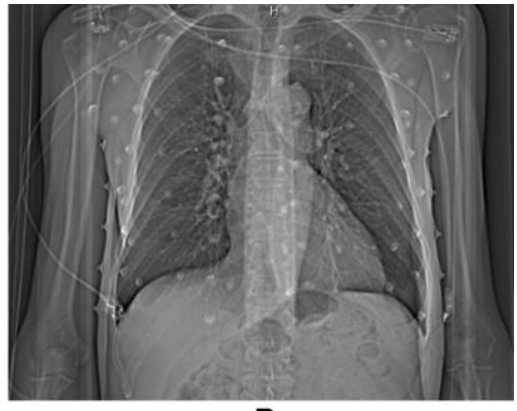

B
Fig.1. Input data for the inverse solution.

A) Multichannel ECG measurement using 96 chest electrodes. B) Anterior-posterior projection of the whole torso CT scan. White dots indicate electrode positions.

After the multichannel ECG recording, the patients with placed ECG electrodes underwent CT scanning of the whole torso (slice thickness $0.3 \mathrm{~mm}$ ) using the Siemens Somatom Definition system (Fig.1.B). From the obtained CT images patient-specific 3D geometries of the torso, lungs, heart atria and ventricles (epi- and endocardial surfaces), aorta and pulmonary artery were created using TomoCon PACS ${ }^{\circledR}$ software [7].

On the next day, EP study and catheter ablation were performed. Patients underwent the procedure under mild conscious sedation with fentanyl. Intravenous isoproterenol was infused as was deemed necessary for the induction of ectopy in patients with infrequent extrasystole in the beginning of the procedure. Catheter ablation was performed using 3-dimensional electroanatomic mapping (CARTO®, Biosense Webster Inc.). Endocardial bipolar and unipolar electrograms were recorded at bandpass filter (30-250 $\mathrm{Hz}$ for bipolar and $0.1-100 \mathrm{~Hz}$ for unipolar) using EP recording system (LabSystem PRO ${ }^{\mathrm{TM}}$, Boston Scientific). The right ventricle (RV) was mapped via standard femoral approach, and the left ventricle (LV) was mapped via a transseptal approach. Intracardiac echocardiography
(Vivid q, GE) was used to guide the transseptal puncture in patients with the PVC origin in the LV. These patients were anticoagulated with unfractionated heparin for activated clotting time of 250 to $300 \mathrm{~s}$.

The target site for the radiofrequency ablation was determined by activation mapping (finding the earliest local activation time preceding the earliest surface QRS) and confirmed by pace mapping (requiring concordance of 11 out of 12 leads between the pace map and the clinical PVCs). After the target site was located, radiofrequency catheter ablation was performed using an open irrigated ablation catheter (Thermocool Navistar, Biosense Webster Inc.) in the power-control mode $(25-40 \mathrm{~W}$, flow 15 $20 \mathrm{ml} / \mathrm{min}$, max. temperature $43^{\circ} \mathrm{C}$ ). Patients were monitored for a minimum of 15 minutes with isoproterenol challenge after the last energy application to ensure complete abatement of the ventricular ectopy.

Positions of the intracardiac interventions were compared with the location of the resulting dipole from the inverse solution. LV was divided into 17 segments according to the American Heart Association recommendations [8]. RVOT was displayed in the superior view and divided into 12 segments as on the clock. Then, the positions of the focus obtained from the EP study and from the inverse solution were assigned to the appropriate segment. The location of the focus obtained from the EP study was determined by a cardiologist and a technician, who performed the EP study. The location of the focus as obtained from the noninvasive mapping was determined by the technicians, who performed the noninvasive assessment. After that, both results were compared.

The inverse solution was based on single dipole model of the cardiac electric generator computed from measured integral BSPMs representing the initial activation during PVC. Integral BSPMs can be generally defined as

$$
i m=\int_{I} \phi(t) d t
$$

where im is integral body surface potential map,

$\phi(t)$ is body surface potential map in time instant $t$, I is examined time interval.

Assuming that the area activated during the examined time interval I from the initial phase of the ventricular activation can be represented by single dipole, the inverse solution in the predefined position in the ventricles can be computed using the equation:

$$
G^{\prime}=\mathbf{B}^{+} i m
$$

where $G^{\prime}$ is dipolar equivalent integral generator,

$\mathbf{B}^{+}$is pseudo-inverse of the transfer matrix $\mathbf{B}$ representing the relation between the equivalent heart generator and potentials on the torso.

Equivalent generator $G^{\prime}$ was computed for all positions in a regular $3 \mathrm{~mm}$ grid throughout the modeled ventricular myocardium. The best position of the equivalent integral generator was selected according to the criterion of minimal value of the relative residual error (RRE) between the input integral BSPM ( $\mathrm{im}$ ) and map ( $\mathrm{gm}$ ) computed from the equivalent integral generator $G^{\prime}$ : 


$$
R R E=\sqrt{\sum_{i=1}^{n}\left(i m_{i}-g m_{i}\right)^{2}} / \sqrt{\sum_{i=1}^{n} i m_{i}^{2}}
$$

where $n$ is the number of points in the maps.

Three torso models with different complexity were constructed from the whole torso CT scan and used in the inverse solutions (Fig.2.): $\mathrm{H}$ - homogeneous torso; I1 inhomogeneous torso with lungs and heart ventricles; I2 inhomogeneous torso with lungs, heart ventricles and atria, aorta (AO) and pulmonary artery (PA). The conductivity of lungs was assumed four times lower than the conductivity of the torso; the conductivity of heart cavities, $\mathrm{AO}$ and PA was assumed three times higher than the conductivity of the torso.

In each patient the inverse solution was computed for five selected ectopic beats and for all three torso models. Standard deviation (SD) of positions of resulting dipoles from the selected PVC beats was evaluated for each of the torso models.

Fisher's test was used to compare dispersions of the locations of resulting dipoles obtained from the inverse solutions using torso models with different inhomogeneities. To evaluate the differences between the inverse results for various torso models, the mean distances between the positions of resulting dipoles for $\mathrm{H}$ and $\mathrm{I} 1$ models, $\mathrm{H}$ and $\mathrm{I} 2$ models, and I1 and I2 models were calculated for each patient.

\section{RESULTS}

All EP studies were without complications. The average duration of the procedure was 153 minutes, and fluoroscopy time was from 5.4 to 19.6 minutes. Acute success (i.e. an absence of PVCs after ablation) was reached in all patients but there was an early recurrence in Pat004. In patients with a focus in the RVOT, the mean prematurity was $37.6 \mathrm{~ms}$ and the pacemaps accordance was present in all patients. In patients with a focus in the $\mathrm{LV}$, the mean prematurity was $39 \mathrm{~ms}$ and the pacemaps accordance was also 12 of 12 .
Locations of the foci obtained from the EP study and positions of the resulting dipoles obtained from inverse solutions for all torso models are presented in Table 1.

Table 1. Positions of the ablation lesions and inverse solutions.

\begin{tabular}{|l|l|l|}
\hline Patient & $\begin{array}{c}\text { Position } \\
\text { of the ablation } \\
\text { lesion }\end{array}$ & $\begin{array}{c}\text { Position } \\
\text { of the inverse solution } \\
\text { with H, I1, I2 models }\end{array}$ \\
\hline Pat004 & septal RVOT & $\begin{array}{l}\text { septal RVOT } \\
\text { posterior RVOT } \\
\text { posterior RVOT }\end{array}$ \\
\hline Pat005 & left lateral RVOT & $\begin{array}{l}\text { basal anterior LV } \\
\text { left lateral RVOT } \\
\text { left lateral RVOT }\end{array}$ \\
\hline Pat006 & basal inferior LV & $\begin{array}{l}\text { basal inferior LV } \\
\text { basal inferior LV (4/5) } \\
\text { basal inferior LV }\end{array}$ \\
\hline Pat007 & septum RVOT & $\begin{array}{l}\text { septal RVOT } \\
\text { anterior septal RVOT } \\
\text { septal RVOT (2/5) } \\
\text { basal anterior LV } \\
\text { basal antero-lateral LV (2/5) } \\
\text { basal anterior LV (4/5) }\end{array}$ \\
\hline Pat009 & basal anterior LV \\
\hline
\end{tabular}

In three patients the PVC focus was located in the RVOT and for the other two patients it was located in the LV. For all patients (except Pat005 with torso model $\mathrm{H}$ ) the location of the PVC focus obtained from the inverse solution was estimated correctly within the particular chamber, independent of the used torso model. The result for Pat005 with torso model $\mathrm{H}$ was also close to the true origin of the PVC, but it was shifted to anterior side of the left ventricle.

SDs of positions of resulting dipoles for all patients and torso models are depicted in Fig.3. The average SD of the positions for all patients was $2.8 \mathrm{~mm}$ (range 0.0 to $7.2 \mathrm{~mm}$ ) with model $\mathrm{H}, 9.9 \mathrm{~mm}$ (range 0.0 to $29.5 \mathrm{~mm}$ ) with model I1 and $8.6 \mathrm{~mm}$ (range 0.0 to $17.2 \mathrm{~mm}$ ) with model I2.

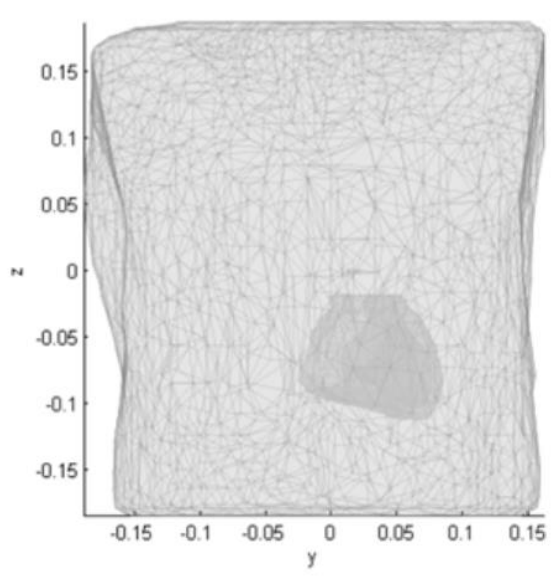

H

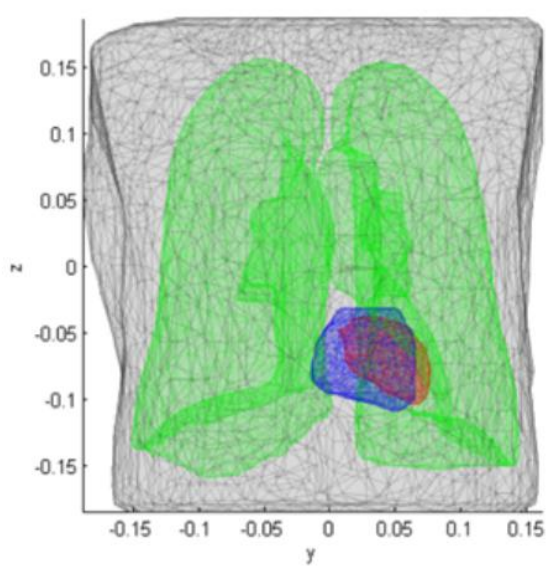

11

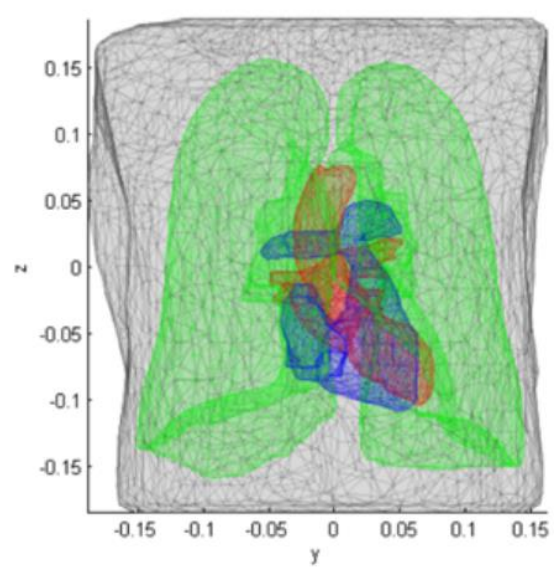

12

Fig.2. Torso models with different inhomogeneities: $\mathrm{H}$ - homogeneous torso; I1 - inhomogeneous torso with lungs and heart ventricles; I 2 - inhomogeneous torso with lungs, heart ventricles and atria, aorta and pulmonary artery. 
Standard deviations of the positions of the resulting dipoles $(\mathrm{mm})$

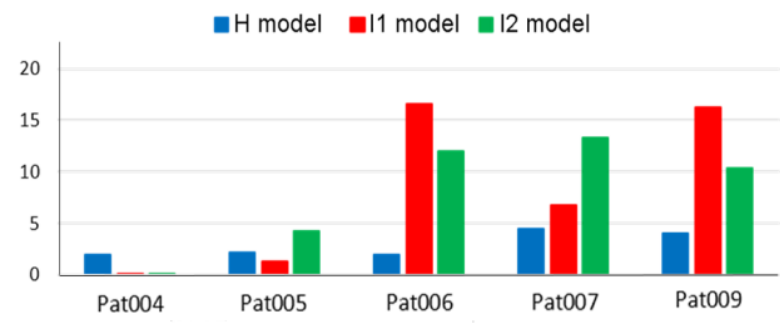

Fig.3. Standard deviations of the positions of resulting dipoles obtained from 5 heart cycles in a homogeneous $(\mathrm{H})$ and two inhomogeneous torso models (I1 and I2) for each patient.

Dispersion of positions of the resulting dipoles obtained with the homogeneous torso model was significantly lower than the dispersions of the inverse results obtained with both inhomogeneous torso models $(\mathrm{p}<0.05)$. Dispersions of dipole positions obtained with inhomogeneous torso models I1 and I2 were not significantly different.

The examples of results of the inverse solution for all three torso models are demonstrated on Pat005 with PVC focus located in the RV (Fig.4.) and on Pat006 with PVC focus located in the LV (Fig.5.).
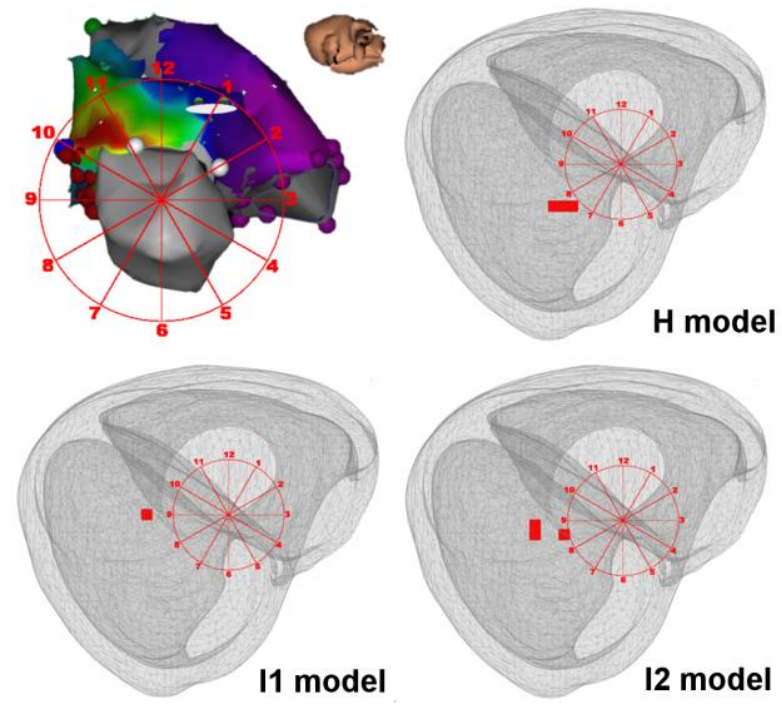

Fig.4. Results for patient Pat005 in superior view. Ablation points from the EP study (upper left) marked by red markers on clock 9 and 10. Results of the inverse solution (marked by red squares) for five selected PVC complexes as obtained with model $\mathrm{H}$ (upper right) and with models I1 and I2 (lower left and right).

In Pat005 the pathological source of the premature ventricular activity was located in the left lateral RVOT during the EP study. The inverse solution with homogeneous torso model localized the position of the PVC focus as slightly shifted to anterior LV. However, inverse solutions with inhomogeneous torso model I1 and I2 showed position close to the EP study location in left lateral RVOT. For all torso models locations of the resulting dipoles for all five ectopic beats were very close to each other (Fig.4.).
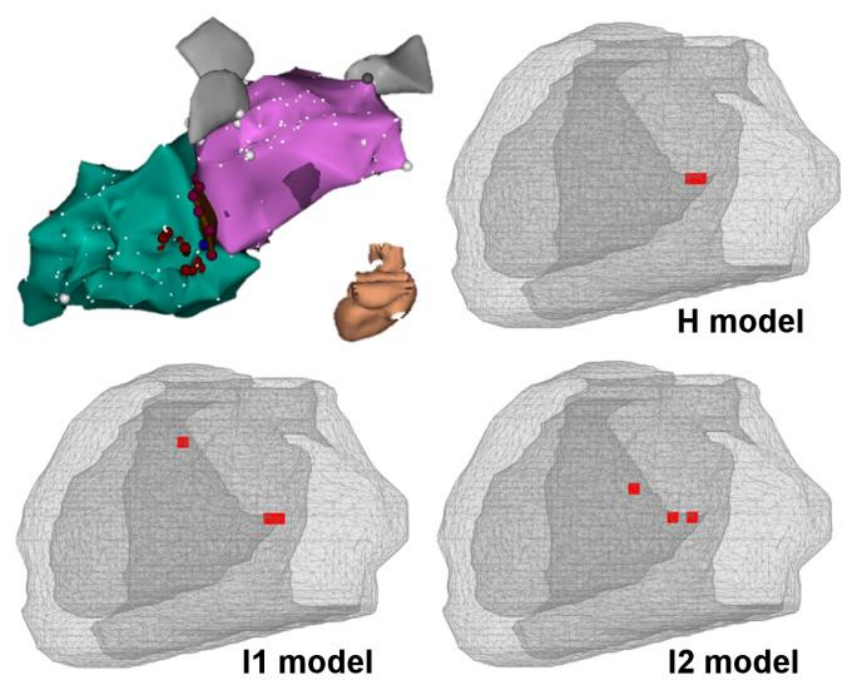

Fig.5. Results for patient Pat006 in posterior view. Ablation points from the EP study (upper left) marked by red dots in the posterior LV. Results of the inverse solution (marked by red squares) for five selected PVC complexes as obtained with model $\mathrm{H}$ (upper right) and models I1 and $\mathrm{I} 2$ (lower left and right).

In Pat006 the focus of the ectopic beat was ablated in basal inferior LV during the EP study. Results of the inverse solution for five selected PVC complexes with torso model $\mathrm{H}$ were located in basal inferior LV. Positions of the resulting dipoles obtained with torso model I1 were in the basal inferior LV with one outlier in basal anterolateral LV causing the highest SD of positions of the resulting dipoles among all patients. Resulting dipoles obtained with torso model I2 were also distributed along the basal inferior part of the LV. Nevertheless, they were in accordance with the results from the EP study (Fig.5.).

Mean distances between the groups of resulting dipoles for the three used torso models are depicted in Fig.6.

Mean distances between the groups of resulting dipoles $(\mathrm{mm})$

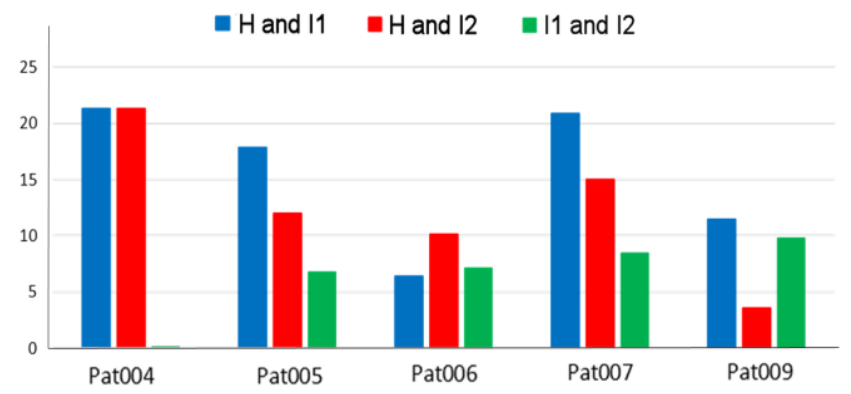

Fig.6. Mean distances between the groups of resulting dipoles for three torso models: $\mathrm{H}$ and $\mathrm{I} 1$ models (blue); $\mathrm{H}$ and $\mathrm{I} 2$ models (red); I1 and 2 models (green).

Largest distances between the above mentioned groups of resulting dipoles were obtained between torso models $\mathrm{H}$ and I1 in all patients, except Pat006. Smallest distances were between torso models I1 and I 2 in all patients with ectopic focus located in the RV (Pat004, Pat005, and Pat007). 


\section{DisCUSSION}

The influence of torso model inhomogeneities on the inverse localization of the focus of ectopic ventricular activity was studied on five PVC patients. Three torso models with different complexity of inhomogeneities were created for each patient and the inverse problem was solved for five ectopic beats selected from the patient's multichannel ECG records. SD of positions of the resulting dipoles was used to estimate the stability of the inverse solution. Minimal SD $(2.8 \mathrm{~mm})$ was obtained when homogeneous torso model $\mathrm{H}$ was used for computation of the inverse solution. This value is comparable with the spatial resolution of the method because predefined dipole positions for the possible inverse results were placed in a $3 \mathrm{~mm}$ grid.

Adding inhomogeneities to the torso model led to increased SD of the positions of the resulting dipoles. However, for some beats more accurate results, better corresponding with anatomical description of the ablation positions during the intracardiac EP study were obtained for inhomogeneous torso models.

SDs of the resulting dipoles obtained with all torso models were bigger in patients with PVC focus located in the LV, which can be caused by the larger distance of the LV position to the measuring electrodes.

Mean distances between the resulting dipoles computed for the three torso models were calculated in order to determine how the positions of resulting dipoles will be shifted if different torso models are used. The biggest distances (from $3.5 \mathrm{~mm}$ to $21.3 \mathrm{~mm}$ ) were between the results obtained with homogeneous torso model $\mathrm{H}$ and both inhomogeneous torso models I1 or I2. The smallest distances (from $0.0 \mathrm{~mm}$ to $9.8 \mathrm{~mm}$ ) were between the groups of resulting dipoles obtained with inhomogeneous torso models. This is probably caused by closer similarity of the two inhomogeneous torso models.

Quantitative evaluation of the distance between the location of the PVC focus from the inverse solution (using the heart model obtained from CT) and the location of the successful ablation (using the heart geometry obtained from the navigation system CARTO®) is not presented because the exact merging of the two geometries has not been performed yet. Therefore, evaluation of the accuracy of the inverse solution is based only on the anatomical description of the positions.

Comparison of the models showed that homogeneous torso model $\mathrm{H}$ provides the smallest dispersion of the inverse results from different ectopic beats and good correspondence with results of the EP study in most cases. On the other hand, despite lower stability expressed by higher dispersion of results from different ectopic beats, most inverse results obtained with the inhomogeneous torso model I2 showed very good correspondence with positions of the ablation points during the EP studies and the remaining results were shifted less than $2 \mathrm{~cm}$.

The results of inverse solutions can be also influenced by other factors: methods for solving the inverse problem, number of measured ECG leads, methods for signal processing and selection of the time interval for integral BSPMs, accuracy of the CT scan segmentation, parameters of the thorax model (conductivities of lungs and blood in heart cavities), etc.

The inverse problem is generally ill-posed and therefore some regularization is needed for its solution. Usually, the Tikhonov regularization method is used. Studies on numerical factors of the inverse solution using the Tikhonov regularization were reported in [9] and [10]. Another approach to solve the inverse problem is to use an equivalent cardiac generator in the form of moving or fixed dipoles [11], [12] that was used also in this study.

The number of the electrodes used for BSPMs varies up to 256 [13]. The amount and positions of BSPM leads required for accurate inverse solution was studied by van der Graaf, Lux and Hoekema in [14], [15], [16]. They showed that if limited number of leads (24 - 64) is used, they should be placed non-uniformly on both, anterior and posterior sides of the torso with highest density on the left anterior chest where the surface electrical potential has the highest gradients. In our study 96 evenly distributed leads on the patient's torso were used for the BSPMs.

It was shown elsewhere that for accurate inverse solution patient-specific geometry should be used [13], [17], [18]. Precision of the reconstruction of the patient-specific geometry from CT images was studied by Prakosa in [19] and its influence on accurate electrophysiological imaging by Rahimi [20]. In this study specialized image-processing software TomoCon PACS $®$ was used to construct precise patient-specific torso geometries from whole torso CT scans.

Impact of the complexity of the patient-specific geometry (organs and their conductivities in the volume conductor model) on surface potentials was studied by Doessel [21] and Zemzemi [22]. Global study of the influence of the torso inhomogeneities on atrial and ventricular ECG signals was performed in [21]. Realistic forward simulations showed that the conductivity of blood in the main vessels had high impact on the atrial signals. Nevertheless, the influence of this conductivity on the inverse solution using ventricular signals was not evaluated in the study. Ramanathan concluded in [23] that in clinical application it is not necessary to include torso inhomogeneities in noninvasive electrophysiological imaging but Zemzemi showed in [22] that using inhomogeneities in combination with low noise ECGs improves the accuracy of the inverse solution. These results are in accordance with the results of our study.

Attempts to find the focus of ectopic ventricular activity by solving the inverse problem were performed on simulated data [24] and also in animal experiments [24], [25], [26]. Few validation studies on patients were published in [13], [27], [28].

This study was performed on five patients with monomorphic PVC and their BSPMs were used as input data for the inverse solution to one dipole. Patient-specific torso geometries were constructed from the whole torso CT scans. Despite the observed dispersion of the resulting dipoles, their noninvasively located positions were accurate enough to define the correct cardiac compartment, in close proximity to the site where the successful ablation procedure was performed. Homogeneous torso model $\mathrm{H}$ and inhomogeneous torso model I2 are preferable for the inverse solution to define the right site of the PVC focus. 
The limitation of the study is that the results are based on a small group of five patients, which can be considered sufficient for testing the method, however, the clinical relevance will require testing this method on a larger group of patients.

\section{CONCLUSIONS}

Five patients with PVC underwent multichannel BSPM and CT scanning. Integral BSPMs from the initial $20 \mathrm{~ms}$ of five ectopic beats were computed for each patient. Patientspecific torso models were created from whole torso CT scans and together with integral BSPMs used as input data for noninvasive localization of the ectopic focus by inverse solution in terms of a single dipole.

One homogeneous and two inhomogeneous torso models were used for evaluation of the impact of inhomogeneities on the inverse solution. The most stable results were obtained with the homogeneous torso model $\mathrm{H}$. Unfortunately, the use of this model might lead to less accurate localization of the PVC focus in some cases. The use of inhomogeneous torso model I2, despite higher dispersion of results from different ectopic beats, led in most cases to very good correspondence of the inverse solution with the result of the EP study and the remaining results were also in close proximity to the location of the ablation lesion. Therefore, torso model I2 should be used to clarify the position of the PVC focus. Comparison of solutions with torso models I1 and I 2 did not show significantly different dispersions but localization of the focus was better when torso model I2 was used.

Although the proposed localization method is relatively time consuming and requires additional BSPM and CT, it is noninvasive and the benefits of its involvement into the preEP study protocol could be reduced time of the EP study, lower load on the patient and increased success rate of the ablation.

For final conclusion, whether the inhomogeneous torso model including lungs, heart ventricles and atria, aorta and pulmonary artery filled with blood can increase the accuracy of the inverse solution, more cases with exact quantitative evaluation need to be studied.

\section{ACKNOWLEDGMENT}

The present study was supported by research grants $3 / 229 /$ OHK 4/3T/17 from the SGS CVUT and PRVOUK P35 in the Czech Republic, APVV-14-0875 from the Research and Development Agency and 2/0071/16 from the VEGA Grant Agency in Slovakia.

\section{REFERENCES}

[1] Bogun, F., Crawford, T., Reich, S., et al. (2007). Radiofrequency ablation of frequent, idiopathic premature ventricular complexes: Comparison with a control group without intervention. Heart Rhythm, 4 (7), 863-867.

[2] Senderek, T., Bednarek, J., Lelakowski, J. (2015). The effectiveness of RF ablation of ventricular ectopic beats made using selected mapping techniques. Polski Merkuriusz Lekarski, 39 (233), 271-276.
[3] Tysler, M., Tinova, M. (1993). Representation of myocardium depolarization by simple models. In Computers in Cardiology 1993, September 5-8, 1993. IEEE, 703-706.

[4] Punshchykova, O., Svehlikova, J., Kneppo, P., Maksymenko, V., Tysler, M. (2014). Noninvasive localization of the ectopic focus using time integral ECG mapping. Experimental and Clinical Cardiology, 20 (7), 1564-1570.

[5] Tysler, M., Svehlikova, J., Punshchykova, O., Kneppo, P., Maksymenko, V. (2015). Noninvasive localization of ectopic ventricular activity using BSPM and different patient torso models. In IEEE 35th International Conference on Electronics and Nanotechnology (ELNANO), April 21-24, 2015, Kyiv, Ukraine. IEEE, 325-329.

[6] Rosík, V., Karas, S., Hebláková, E., Tyšler, M., Filipová, S. (2007). Portable device for high resolution ECG mapping. Measurement Science Review, 7 (6), 57-61.

[7] TatraMed Software s.r.o. (2016). TomoCon PACS. http://www.tatramed.sk/pacsItem?element=3\&parentI $\mathrm{d}=18 \&$ type $=19$

[8] Cerqueira, M.D., Weissman, N.J., Dilsizian, V., Jacobs, A.K., Kaul, S., Laskey, W.K., et al. (2002). Standardized myocardial segmentation and nomenclature for tomographic imaging of the heart: A statement for healthcare professionals from the cardiac imaging committee of the council on clinical cardiology of the American Heart Association. Circulation, 105 (4), 539-542.

[9] Barnes, J.P., Johnston, P. (2016). Application of robust Generalised Cross-Validation to the inverse problem of electrocardiology. Computers in Biology and Medicine, 69, 213-225.

[10] Tuboly, G., Kozmann, G., Maros, I. (2015). Computational aspects of electrocardiological inverse solutions. IFAC-PapersOnLine, 48 (20), 48-51.

[11] Lai, D., Liu, Ch., Eggen, M.D., Iazzo, P., He, B. (2010). Equivalent moving dipole localization of cardiac ectopic activity in a swine model during pacing. IEEE Transactions on Information Technology in Biomedicine, 14 (6), 1318-1326.

[12] Xanthis, V.P., Bonovas, M., Kyriacou, G.A. (2007). Inverse problem of ECG for different equivalent cardiac sources. Piers Online, 3 (8), 1222-1227.

[13] Cluitmans, M.J.M., Peeters, R.L.M., Westra, R.L., Volders, P.G.A. (2015). Noninvasive reconstruction of cardiac electrical activity: Update on current methods, applications and challenges. Netherlands Heart Journal, 23 (6), 301-311.

[14] van der Graaf, A.W.M., Bhagirath, P., de Hooge, J., de Groot, N.M.S., Gotte, M.J.W. (2016). A priori model independent inverse potential mapping: The impact of electrode positioning. Clinical Research in Cardiology, 105, 79-88. 
[15] Lux, R.L., Smith, R.F., Abildskov, J.A. (1978). Limited lead selection for estimating body surface potentials in electrocardiography. IEEE Transactions on Biomedical Engineering, 25 (3), 270-276.

[16] Hoekema, R., Uijen, G.J., van Oosterom, A. (1999). On selecting a body surface mapping procedure. Journal of Electrocardiology, 32 (2), 93-101.

[17] Cheng, L.K., Sands, G.B., Pullan, A.J. (2005). Construction of patient specific geometries suitable for the inverse problem of electrocardiography. In 27th Annual International Conference of the IEEE Engineering in Medicine and Biology Society (IEEEEMBS 2005), January 17-18, 2005. IEEE, 7201-7203.

[18] Tysler, M., Svehlikova, K., Punshchykova, O., Lenkova, J. (2013). Influence of torso model accuracy on the noninvasive localization of heart pathologies. Acta Mechanica Slovaca, 17 (3), 18-25.

[19] Prakosa, A., Malamas, P., Zhang, S., Pashakhanloo, F., Arevalo, H., Herzka, D.A., et al. (2014). Methodology for image-based reconstruction of ventricular geometry for patient-specific modeling of cardiac electrophysiology. Progress in Biophysics \& Molecular Biology, 115 (2-3), 226-234.

[20] Rahimi, A., Wang, L. (2015). Sensitivity of noninvasive cardiac electrophysiological imaging to variations in personalized anatomical modeling. IEEE Transactions on Biomedical Engineering, 62 (6), 1563-1575.

[21] Keller, D.U.J., Weber, F.M., Seemann, G., Doessel, O. (2010). Ranking the influence of tissue conductivities on forward-calculated ECGs. IEEE Transactions on Biomedical Engineering, 57 (7), 1568-1576.

[22] Zemzemi, N., Dobrzynski, C., Bear, L., Potse, M., Dallet, C., Coudiére, Y., Dubois, R., Duchateau, J. (2015). Effect of the torso conductivity heterogeneities on the ECGI inverse problem solution. In Computing in Cardiology 2015, September 6-9, 2015. IEEE, 233236.
[23] Ramanathan, C., Rudy, Y. (2011). Electrocardiographic imaging: II. Effect of torso inhomogeneities on noninvasive reconstruction of epicardial potentials, electrograms, and isochrones. Journal of Cardiovascular Electrophysiology, 12 (2), 241-252.

[24] Ghodrati, A., Brooks, D.H., Tadmor, G., MacLeod, R.S. (2006). Wavefront-based models for inverse electrocardiography. IEEE Transactions on Biomedical Engineering, 53 (9), 1821-1831.

[25] Liu, C., Skadsberg, N.D., Ahlberg, S.E., Swingen, C.M., Iaizzo, P.A., He, B. (2008). Estimation of global ventricular activation sequences by noninvasive 3dimensional electrical imaging: Validation studies in a swine model during pacing. Journal of Cardiovascular Electrophysiology, 19 (5), 535-540.

[26] Liu, C., Zhang, X., Liu, Z., Pogwizd, S.M., He, B. (2006). Three-dimensional myocardial activation imaging in a rabbit model. IEEE Transactions on Biomedical Engineering, 53, 1813-1820.

[27] Št'ovíček, P., Havránek, Š., Šimek, J., Zborník, M., Mlček, M., Kittnar, O. (2010) Isopotential ECG imaging correctly identified endocardial ectopic activation site in the case of arrhythmia from right ventricular outflow tract. IFMBE Proceedings, 25 (4), 1965-1968.

[28] Coll-Font, J., Erem, B., Stovicek, P., Brooks, D.H., van Dam, P.M. (2015). Quantitative comparison of two cardiac electrical imaging methods to localize pacing sites. Computing in Cardiology 2015, September 6-9, 2015. IEEE, 217-220.

Received January 28, 2016. Accepted April 11, 2016 